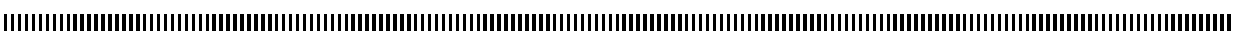
|

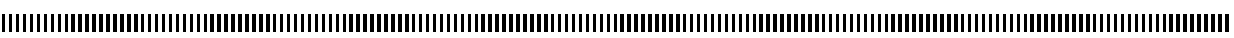

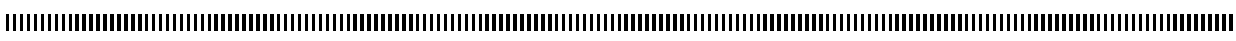

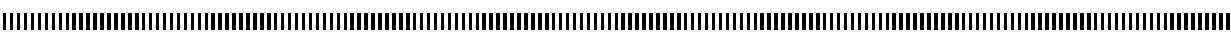

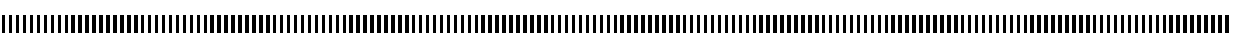

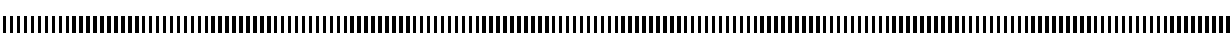

\title{
Integer calculus on the Harthong-Reeb Line
}

\author{
Wallet Guy \\ Laboratoire de Mathématiques, Image et Applications \\ Université de La Rochelle \\ Avenue Michel Crépeau \\ 17042 La Rochelle France \\ guy.wallet@univ-lr.fr
}

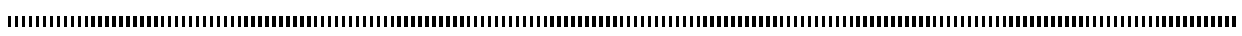

ABSTRACT. In this work, we give a presentation of the so-called Harthong-Reeb line. Only based on integer numbers, this numerical system has the striking property to be roughly equivalent to the continuous real line. Its definition requires the use of a natural number $\omega$ which is infinitely large in the meaning of nonstandard analysis. Following the idea of G. Reeb, we show how to implement in this framework the Euler scheme. Then we get an exact representation in the Harthong-Reeb line of many real functions like the exponential. Since this representation is given with the help of an explicit algorithm, it is natural to wonder about the global constructivity of this numerical system. In the conclusion, we discuss this last point and we outline some new directions for getting analogous systems which would be more constructive.

RÉSUMÉ. Dans ce travail, nous donnons une présentation de la droite dite d'Harthong-Reeb. II s'agit d'un système numérique uniquement basé sur les nombres entiers et dont la propriété frappante est qu'il est à peu près équivalent à la droite réelle continue. Sa définition nécessite l'utilisation d'un nombre naturel $\omega$ qui est infiniment grand au sens de l'analyse nonstandard. Suivant l'idée de G. Reeb, nous montrons comment on peut implémenter le schéma d'Euler dans ce cadre. Alors, on obtient une représentation exacte dans la droite d'Harthong-Reeb de nombreuses fonctions réelles comme la fonction exponentielle. Puisque cette représentation est donnée au moyen d'un algorithme explicite, il est naturel de s'interroger sur la constructivité globale de ce système numérique. Dans la conclusion, nous discutons ce dernier point et nous esquissons de nouvelles directions pour obtenir des systèmes analogues dotés d'une meilleure constructivité.

KEYWORDS : nonstandard analysis, arithmetization, Euler scheme, discrete line, constructive mathematics.

MOTS-CLÉS : analyse nonstandard, arithmétisation, schéma d'Euler, droite discrète, mathématiques constructives.

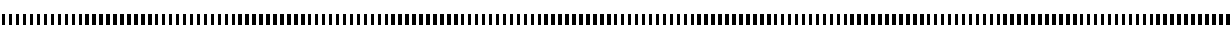




\section{Introduction}

The first goal of this paper is to give an idea of some of the works which have been done, mainly during the eighties and the first half of the nineties, in a part of the french school of nonstandard analysis on the matter of computation in analysis with integers numbers using the framework of the so called Harthong-Reeb line. The initiators of this theme are undoubtedly G. Reeb and J. Harthong. These two leading figures wanted to define and to experiment a new way of designing the mathematical continuum with the strong feature of using only integer numbers $[14,15]$. This project was partly based on a philosophical analysis which is not fully developed here [16, 32, 22, 41]. In addition to G. Reeb and J. Harthong, the main protagonists were J.P. Reveillès, A. Troesch, E. Urlacher, M. Diener and H. Holin [36, 37, 38, 34, 10, 17]. For the most part, our presentation will follow the works of the preceding authors. However, we have added some explanations, terminologies or developments in order to clarify certain points. On the other hand, we do not claim to reflect all the works which have been done in this direction.

The second goal of this study is to show that the scientific interest of this topic is still relevant. Actually, the Harthong-Reeb line is a transition space between the discret line $\mathbb{Z}$ and the continuous line $\mathbb{R}$. Thus, this space is a kind of paradigm which occupies an important place in the context of a general study of scaling transformations. Moreover, the Harthong-Reeb line is also an alternative theory of the real line with the striking property that the basic elements of this space are integers numbers. Since computations are easy and sure with integers, an interesting question is to measure to what extent this concept is really constructive. At the frontier of computer science and mathematics, a research team composed of E. Andres, L. Fuchs and G. Largeteau from Poitiers University, A. Chollet and the author from La Rochelle University is currently working on this issue [13]. The present paper is also a consequence of this collective work.

The basic remark leading to the Harthong-Reeb line is that the discret space $\mathbb{Z}$ seen from afar looks like the real line $\mathbb{R}$. Forcefully expressed by J. Harthong ${ }^{1}$, this point of view gave birth during the eighties to a scientific program about a new representation of a continuous line. The main idea is that it is sufficient to perform a strong metric contraction on the arithmetic of integers to get a numerical system which is equivalent to the system of real numbers. The expected benefits are: 1) safety and speed for computation on integers without using uncontrolled floating numbers, 2) the meeting of discret and continuous aspects in a single space.

Obviously, this program is based on the use of nonstandard analysis (NSA). Indeed, it is well known ${ }^{2}$ that, when dealing with a problem involving real numbers, one of the most significant contributions of NSA is the fruitful possibility of translating the continuous initial problem into a discrete and finitely presented one. However, there is a high specificity of the program of the Harthong-Reeb line relative to the usual practice of NSA:

- the purpose is not to use a finite discretization as an auxiliary tool for resolving a continuous problem but to replace completely classical real numbers by integers;

- consequently, only a minimal form of nonstandard analysis is sufficient.

1. $\mathbb{R}$, c'est $\mathbb{Z}$ vu de loin.

2. For those who have really tried to see what is nonstandard analysis. 


\section{A Minimal Form of Nonstandard Analysis}

Among the different presentations of nonstandard analysis, the people close to Reeb has generally preferred the axiomatic version of Internal Set Theory due to E. Nelson [28]. One advantage of this approach is that it is possible to weaken the nonstandard addition in order to get simple axioms perfectly suited to some purpose. That is what we do in this study. In the spirit of some works of Nelson or Lutz [29, 23], we perform a syntactic forcing of the theory of integer numbers by introducing a new predicate $\mathrm{lim}$ on elements of $\mathbb{N}$ and $\mathbb{Z}: \lim (X)$ reads "the number $X$ is limited". This predicate is external to the usual theory of integer numbers and its ultimate meaning derives from the following rules.

LIM1. The numbers 1 is limited.

LIM2. The sum and the product of two limited numbers are limited.

LIM3. There are non limited integer numbers.

LIM4. For all $(X, Y) \in \mathbb{Z}^{2}$, if $X$ is limited and $|Y| \leq|X|$, then $Y$ is limited.

For reading conveniences, we introduce the following notations:

- $\forall{ }^{\lim } X F(X)$ is an abbreviation for $\forall X(\lim (X) \Rightarrow F(X)$ which can be read as "for all limited $X$, we have $F(x)$ ".

$-\exists^{\lim } X F(X)$ is an abbreviation for $\exists X(\lim (X) \wedge F(X)$ which can be read as "exists a limited $X$ such that $F(X)$ ".

Here we have to insist on the fact that these rules are added to every classical property (axioms or theorems) over integer numbers. Everything that was classically true remains true. We simply improve the language by a syntactic enrichment. These rules imply that $\mathbb{N}$ splits into two classes: the class $\mathbb{N}_{\text {lim }}:=\{0,1, \ldots\}$ of natural limited numbers (stable by arithmetical operations), and the class $\mathbb{N}_{+\infty}$ of natural non limited numbers. Moreover, a non limited natural number is bigger than every limited natural number. Thus, the non limited natural numbers are said infinitely large. In the same way, the set $\mathbb{Z}$ splits into three classes: the class $\mathbb{Z}_{l i m}$ of limited integers, the class $\mathbb{Z}_{+\infty}:=\mathbb{N}_{+\infty}$ and the class $\mathbb{Z}_{-\infty}:=-\mathbb{N}_{+\infty}$. In the following figure, $\omega$ denotes an arbitrary infinitely large natural number.

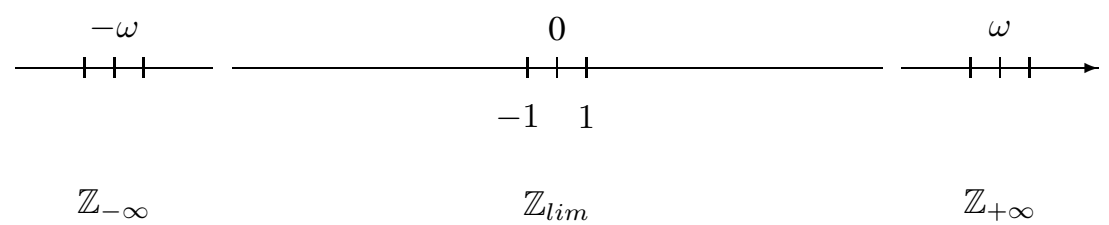

Figure 1. Different orders of magnitude on $\mathbb{Z}$

Lets us add some technical but important remarks. A mathematical property or formula which does not contains the new predicate $\mathrm{lim}$ is called an internal formula. For 
example, the formula $X+1>X$ is internal. An internal formula is therefore a classical formula on numbers. In contrast, an external formula uses explicitly the new predicate $\lim$; for example, $\lim (X)$ or $\forall^{\lim } X, Y<X$ are external formulae. Since everything that was true remains true, for all internal formula $\mathcal{P}(X)$, we can build the set $P=\{X \in \mathbb{N} ; \mathcal{P}(X)\}$ which possesses the classical properties of subsets of $\mathbb{N}$; for example, if the set $P$ is not empty and bounded above, then $P$ has a maximal element. This is no longer true for an external property; for instance, if we consider the external property $\lim (X)$, the collection $\mathbb{N}_{\text {lim }}=\{X \in \mathbb{N} ; \lim (X)\}$ of limited natural numbers is a non empty and bounded above; nevertheless, this collection cannot have a bigger element since $X+1$ is limited for all limited $X$. A collection of numbers defined by an external property which cannot be a set of numbers in the classical meaning is called external set. Hence, $\mathbb{N}_{\text {lim }}$ is an external part of $\mathbb{N}$. The radical difference between internal and external properties is not just a formal limitation because it gives birth to a new mathematical tool called the overspill principle.

Proposition 1. (Overspill principle) Let $\mathcal{P}(x)$ be an internal formula such that $\mathcal{P}(n)$ is true for all limited $n \in \mathbb{N}$. Then, there exists an infinitely large $\nu \in \mathbb{N}$ such that $\mathcal{P}(m)$ is true for all natural numbers $m$ such that $0 \leq m \leq \nu$.

Proof. The collection $A=\{X \in \mathbb{N} ; \forall Y \in[0, X] \mathcal{P}(Y)\}$ is a internal set (i.e. a classical set) containing $\mathbb{N}_{\text {lim }}$. Since $\mathbb{N}_{\text {lim }}$ is an external set, the inclusion $\mathbb{N}_{\text {lim }} \subset A$ is strict and leads to the result.

In the same way, the application of an inductive reasoning on an external formula can be illegitimate. For instance, 0 is limited and $X+1$ is limited for all limited $X \in \mathbb{N}$. Nevertheless, we see from (LIM3) that not all integers are limited. To improve the power of our nonstandard tool, it is necessary to add a special principle for external induction. Nevertheless, a feature of the works we are going to present is that we do not really need this kind of new rule ${ }^{3}$. Accordingly, we will only use the four axioms LIM1, LIM2, LIM3 and LIM4. The last rule will be laid down in an appendix.

\section{Exporting the different scales on $\mathbb{R}$}

Thanks to the predicate lim, we can define some new scale concepts on $\mathbb{R}$.

Definition 1. Let $x$ be real number. We say that:

1) $x$ is limited in case $\exists^{\text {lim }} n \in \mathbb{N} \quad|x| \leq n$;

2) $x$ is infinitely large in case $\forall^{l i m} n \in \mathbb{N} \quad n \leq|x|$.

Notation: $x \simeq \infty(x \simeq+\infty$ if $x>0$ and $x \simeq-\infty$ if $x<0)$;

3) $x$ is infinitely small in case $\forall^{\text {lim }} n \in \mathbb{N} \backslash\{0\} \quad|w| \leq \frac{1}{n}$.

Notation: $x \simeq 0$;

4) $x$ is appreciable in case $x$ is neither infinitely small nor infinitely large.

As a consequence, 0 is infinitely small, 1 is appreciable and we have the following immediate properties:

3. A rule on external induction is useful for convergence or completeness properties. For instance, we need this tool in [13] in a proof about the constructive least uper-bound principle. 


$$
\begin{aligned}
& -x \text { appreciable } \Leftrightarrow \exists^{\text {lim }} k \in \mathbb{N} \backslash\{0\} \frac{1}{k} \leq x \leq k ; \\
& -x \simeq 0 \Leftrightarrow x=0 \text { or } x^{-1} \simeq \infty ; \\
& -x \simeq 0 \text { and }\left|x^{\prime}\right| \leq|x| \Rightarrow x^{\prime} \simeq 0 ; \\
& -x \simeq \infty \text { and }|x| \leq\left|x^{\prime}\right| \Rightarrow x^{\prime} \simeq \infty ; \\
& -x \text { limited and }\left|x^{\prime}\right| \leq|x| \Rightarrow x^{\prime} \text { limited; } \\
& -x \simeq 0 \text { and } y \text { appreciable and } z \simeq \infty \Rightarrow|x| \leq|y| \leq|z| .
\end{aligned}
$$

Moreover, if $\mathcal{I}_{s m}$ denotes an arbitrary infinitely small number, $\mathcal{A}_{p p}$ an arbitrary appreciable number, $\mathcal{L}_{i m}$ an arbitrary limited number and $\mathcal{I}_{l a}$ an arbitrary infinitely large number, we have the following symbolic algebra:

$$
\begin{aligned}
& \mathcal{I}_{s m}+\mathcal{I}_{s m}=\mathcal{I}_{s m} \quad \mathcal{I}_{s m}+\mathcal{A}_{p p}=\mathcal{A}_{p p} \quad \mathcal{I}_{s m}+\mathcal{I}_{l a}=\mathcal{I}_{l a} \\
& \mathcal{A}_{p p}+\mathcal{A}_{p p}=\mathcal{L}_{i m} \quad \mathcal{A}_{p p}+\mathcal{I}_{l a}=\mathcal{I}_{l a} \quad \mathcal{I}_{l a}+\mathcal{I}_{l a}=? \\
& \mathcal{I}_{s m} \times \mathcal{I}_{s m}=\mathcal{I}_{s m} \quad \mathcal{I}_{s m} \times \mathcal{A}_{p p}=\mathcal{I}_{s m} \quad \mathcal{I}_{s m} \times \mathcal{I}_{l a}=? \\
& \mathcal{A}_{p p} \times \mathcal{A}_{p p}=\mathcal{A}_{p p} \quad \mathcal{A}_{p p} \times \mathcal{I}_{l a}=\mathcal{I}_{l a}
\end{aligned}
$$

Definition 2. Given $x, y \in \mathbb{R}$, we say that they are infinitely close and we note $x \simeq y$ in case $x-y \simeq 0$.

It is clear that this binary relation $\simeq$ is an equivalence relation on $\mathbb{R}$. For $x \in \mathbb{R}$, its equivalence class for $\simeq$ is the external set $\operatorname{Hal}(x):=\{y \in \mathbb{R} ; x \simeq y\}$ also called the halo of $x$.

We also introduce the external set of limited real numbers

$$
\mathbb{R}_{\text {lim }}:=\{x \in \mathbb{R} ; x \text { limited }\}
$$

and the numerical system of limited real numbers $\left(\mathbb{R}_{\text {lim }}, \simeq, \lesssim,+, \times, 0,1\right)$ where the relation $\lesssim$ is defined by

$$
x \lesssim y \quad \Longleftrightarrow \quad x \leq y \text { or } x \simeq y
$$

\section{The Harthong-Reeb line as a numerical system}

For $x \in \mathbb{R}$, let $\lfloor x\rfloor$ be the integer part of $x$ defined by

$$
\lfloor x\rfloor \in \mathbb{Z} \text { and }\lfloor x\rfloor \leq x<\lfloor x\rfloor+1
$$

and let $\{x\}:=x-\lfloor x\rfloor$ be the non integer part of $x$, so that

$$
0 \leq\{x\}<1 \text { and } x=\lfloor x\rfloor+\{x\}
$$

Once and for all in this section, we choose an element $\omega \in \mathbb{N}$ such that $\omega \simeq+\infty$. Now, our purpose is to define a new numerical sytem equivalent to the preceding one $\left(\mathbb{R}_{\text {lim }}, \simeq, \leq,+, \times, 0,1\right)$ but such that all the elements are integers and in which $\omega$ is the new unit: the Harthong-Reeb line. Firstly, we will introduce the underlying set $\mathcal{H} \mathcal{R}_{\omega}$, secondly we will define the equality $=_{\omega}$, the order relation $\leq_{\omega}$ and finally the algebraic structure $\left(+\omega, \times_{\omega}\right)$. We have chosen a relatively formal presentation of the HarthongReeb line ${ }^{4}$ which is close to the one given by M. Diener [10].

4. Much more formal that the usual style of Reeb and Harthong. 
Definition 3. The set of integers limited at the scale $\omega$ is

$$
\mathcal{H} \mathcal{R}_{\omega}:=\left\{X \in \mathbb{Z} ; \exists^{l i m} n \in \mathbb{N} \quad|X| \leq n \omega\right\}
$$

It is clear that $\mathcal{H} \mathcal{R}_{\omega}$ is an external subset of $\mathbb{Z}$.

Definition 4. On the set $\mathcal{H} \mathcal{R}_{\omega}$, we introduce the two following binary relations:

- the equality at the scale $\omega$ defined by

$$
\forall X, Y \in \mathcal{H} \mathcal{R}_{\omega} \quad X={ }_{\omega} Y \quad \Longleftrightarrow \quad \forall^{l i m} n \in \mathbb{N} \quad n|X-Y| \leq \omega ;
$$

- the order relation at the scale $\omega$ defined by

$$
\forall X, Y \in \mathcal{H R}_{\omega} \quad X \leq_{\omega} Y \quad \Longleftrightarrow \quad X \leq Y \text { or } X={ }_{\omega} Y .
$$

Proposition 2. The relation ${ }_{\omega}{ }_{\omega}$ is an equivalence relation on $\mathcal{H} \mathcal{R}_{\omega}$.

Proof. We just show that $=_{\omega}$ is transitive. Let $X, Y$ and $Z \in \mathcal{H} \mathcal{R}_{\omega}$ such that $X={ }_{\omega} Y$ and $Y={ }_{\omega} Z$. Then, for every limited $n \in \mathbb{N}$

$$
n|X-Y| \leq \omega \text { and } n|Y-Z| \leq \omega
$$

thus $n|X-Z| \leq 2 \omega$. Taking $n=2 m$, we get

$$
\forall^{l i m} m \in \mathbb{N} \quad m|X-Z| \leq \omega .
$$

Proposition 3. For all $X, Y \in \mathcal{H R}_{\omega}$, we have $X \leq_{\omega} Y$ if and only if

$$
\forall^{l i m} n \in \mathbb{N} \quad n(X-Y) \leq \omega
$$

Proof. Suppose that $X \leq_{\omega} Y$, that is to say $X \leq Y$ or $X=_{\omega} Y$. If $X \leq Y$, then for all limited $n \in \mathbb{N}$ we have $n(X-Y) \leq 0 \leq \omega$ and if $X={ }_{\omega} Y$, then for all limited $n \in \mathbb{N}$ we have $n(X-Y) \leq n|X-Y| \leq \omega$.

Suppose now that $n(X-Y) \leq \omega$ for all limited $n \in \mathbb{N}$. Then, we have $X \leq Y$ or $X>Y$ and this last case, $X-Y=|X-Y|$ and thus $n|X-Y| \leq \omega$ for each limited $n \in \mathbb{N}$.

Proposition 4. The relation $\leq_{\omega}$ is an order relation relatively to the equality $={ }_{\omega}$ at the scale $\omega$, that is to say:

(1) $\forall X \in \mathcal{H} \mathcal{R}_{\omega} \quad X \leq_{\omega} X$;

(2) $\forall X, Y \in \mathcal{H \mathcal { R }} \omega \quad X \leq_{\omega} Y$ and $Y \leq_{\omega} X \Rightarrow X={ }_{\omega} Y$;

(3) $\forall X, Y, Z \in \mathcal{H R}_{\omega} \quad X \leq_{\omega} Y$ and $Y \leq_{\omega} Z \Rightarrow X \leq_{\omega} Y$.

Proof. We just give the proof of (3). From the hypothesis, we know that, for every limited $n \in \mathbb{N}$, we have $n(X-Y) \leq \omega$ and $n(Y-Z) \leq \omega$, thus $n(X-Z) \leq 2 \omega$. Taking $n=2 m$, we get $m(X-Z) \leq \omega$ for every limited $m \in \mathbb{N}$. 
Remark Usually, when we have an equivalence relation such as $={ }_{\omega}$ on $\mathcal{H} \mathcal{R}_{\omega}$ or $\simeq$ on $\mathbb{R}_{\text {lim }}$, we consider the associated quotient set in order to replace the initial equivalence relation by the absolute equality on the equivalence classes. It turns out that this usage is not so good and that it is technically simpler to keep the initial equivalence relation. Common in constructive mathematics and theoretical computer sciences, this point of view avoids to create too much new objects. Moreover, in our situation, we are dealing with external sets and the meaning of the associated quotient sets is not clear.

We are now going to introduce the pertinent algebraic operations on $\mathcal{H} \mathcal{R}_{\omega}$.

Definition 5. The sum at the scale $\omega$ is the operation $+{ }_{\omega}$ on $\mathcal{H} \mathcal{R}_{\omega}$ identical to the restriction of the usual sum in $\mathbb{Z}$

$$
\forall X, Y \in \mathcal{H R}_{\omega} \quad X+{ }_{\omega} Y:=X+Y .
$$

The multiplication at the scale $\omega$ is the operation $\times_{\omega}$ on $\mathcal{H R}_{\omega}$ such that

$$
\forall X, Y \in \mathcal{H R}_{\omega} \quad X \times_{\omega} Y:=\left\lfloor\frac{X Y}{\omega}\right\rfloor .
$$

Let us also introduce the following notations:

$-0_{\omega}:=0$ and $1_{\omega}:=\omega$ (both belonging to $\mathcal{H} \mathcal{R}_{\omega}$ );

- For each $X \in \mathcal{H} \mathcal{R}_{\omega} \quad-{ }_{\omega} X:=-X$;

- For each $X \in \mathcal{H R}_{\omega}$ such that $X \neq_{\omega} 0_{\omega} \quad X^{(-1)_{\omega}}:=\left\lfloor\frac{\omega^{2}}{X}\right\rfloor$.

Then, we get the following algebraic properties.

Proposition 5. For every $X, Y, Z \in \mathcal{H} \mathcal{R}_{\omega}$, we have:

(1) $X+{ }_{\omega} Y \in \mathcal{H} \mathcal{R}_{\omega}$ and $X \times_{\omega} Y \in \mathcal{H} \mathcal{R}_{\omega}$;

(2) $-{ }_{\omega} X \in \mathcal{H R}_{\omega}$ and $X^{(-1)_{\omega}} \in \mathcal{H} \mathcal{R}_{\omega}$ if $X \neq{ }_{\omega} 0_{\omega}$;

(3) $X+{ }_{\omega} Y=Y+{ }_{\omega} X$ and $X \times{ }_{\omega} Y=Y \times{ }_{\omega} X$;

(4) $\left(X+{ }_{\omega} Y\right)+{ }_{\omega} Z=X+{ }_{\omega}\left(Y+_{\omega} Z\right)$ and $\left(X \times_{\omega} Y\right) \times{ }_{\omega} Z=_{\omega} X \times_{\omega}\left(Y \times_{\omega} Z\right)$;

(5) $X+{ }_{\omega} 0_{\omega}=X$ and $X \times 1_{\omega}=X$;

(6) $X+{ }_{\omega}-{ }_{\omega} X=0_{\omega}$ and $X \times{ }_{\omega} X^{(-1)_{\omega}}={ }_{\omega} 1_{\omega}$.

Proof. Regarding the sum operation, these properties are trivial. For the product operation, we just have to use systematically the decomposition of any rational number into the sum of its integer part and its fractional part. For instance, let us consider the justification of the point (4) for the multiplication.

From the definition, we get $\left(X \times{ }_{\omega} Y\right) \times{ }_{\omega} Z=\left\lfloor\left\lfloor\frac{X . Y}{\omega}\right\rfloor \frac{Z}{\omega}\right\rfloor$. Using several times the decomposition $U=\lfloor U\rfloor-\{U\}$ with $0 \leq\lfloor U\rfloor<1$, we obtain

$$
\left(X \times{ }_{\omega} Y\right) \times{ }_{\omega} Z=\left\lfloor\frac{X Y Z}{\omega^{2}}\right\rfloor+\left\{\frac{X Y Z}{\omega^{2}}\right\}-\left\{\frac{X Y}{\omega}\right\} \frac{Z}{\omega}-\left\{\left\lfloor\frac{X . Y}{\omega}\right\rfloor \frac{Z}{\omega}\right\}
$$


Since $Z \in \mathcal{H} \mathcal{R}_{\omega}$, there is a limited $n \in \mathbb{N}$ such that $|Z| \leq n \omega$. Hence, we have

$$
\left|\left\{\frac{X Y Z}{\omega^{2}}\right\}-\left\{\frac{X Y}{\omega}\right\} \frac{Z}{\omega}-\left\{\left\lfloor\frac{X . Y}{\omega}\right\rfloor \frac{Z}{\omega}\right\}\right| \leq n+2 .
$$

Since $n+2={ }_{\omega} 0$, we obtain $\left.\left(X \times_{\omega} Y\right) \times_{\omega} Z\right)={ }_{\omega}\left\lfloor\frac{X Y Z}{\omega^{2}}\right\rfloor$. It is clear that a similar treatment would give $X \times{ }_{\omega}\left(Y \times_{\omega} Z\right)={ }_{\omega}\left\lfloor\frac{X Y Z}{\omega^{2}}\right\rfloor$.

It is easy to see that the relation $\leq_{\omega}$, the operations $+_{\omega}, \times_{\omega}$ and the applications $X \mapsto-{ }_{\omega} X$ and $X \mapsto X^{(-1)_{\omega}}$ (for $X \neq{ }_{\omega} 0_{\omega}$ ) are extensional relatively to the equality $={ }_{\omega}$. That means that, for $X, X^{\prime}, Y, Y^{\prime} \in \mathcal{H} \mathcal{R}_{\omega}$ such that $X={ }_{\omega} X^{\prime}$ and $Y={ }_{\omega} Y^{\prime}$, we have

$$
\begin{aligned}
& -X \leq_{\omega} Y \quad \Rightarrow \quad X^{\prime} \leq_{\omega} Y^{\prime} ; \\
& -X+{ }_{\omega} Y={ }_{\omega} X^{\prime}+{ }_{\omega} Y^{\prime} \text { and } X \times_{\omega} Y={ }_{\omega} X^{\prime} \times{ }_{\omega} Y^{\prime} ; \\
& --\omega X={ }_{\omega} X^{\prime} \text { and } X^{(-1)_{\omega}}={ }_{\omega} X^{\prime(-1))_{\omega}}\left(\text { for } X \neq{ }_{\omega} 0_{\omega}\right) .
\end{aligned}
$$

It is now the time to give our formal definition of the Harthong-Reeb line.

Definition 6. The numerical system $\left(\mathcal{H} \mathcal{R}_{\omega},=_{\omega}, \leq_{\omega},+{ }_{\omega}, \times_{\omega}\right)$ is called the HarthongReeb line.
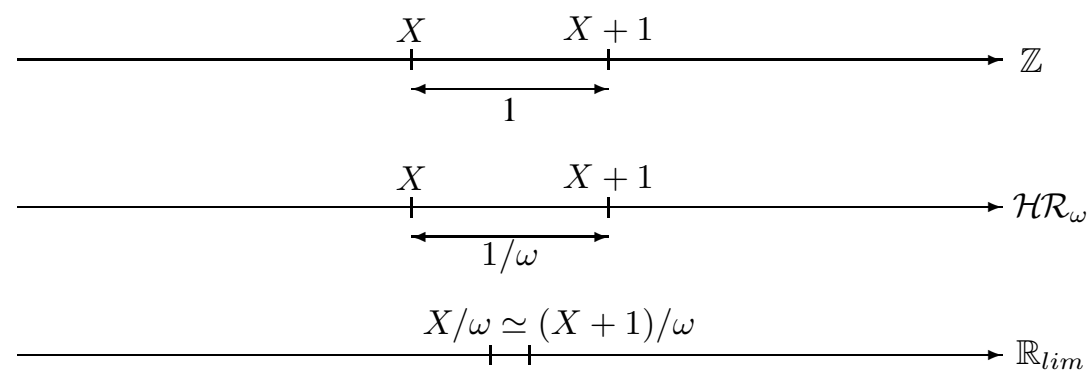

Figure 2. $\mathbb{Z}, \mathcal{H} \mathcal{R}_{\omega}$ and $\mathbb{R}_{\text {lim }}$

That concept explicitly depends on a parameter $\omega \in \mathbb{N}$ such that $\omega \simeq+\infty$. Although the elements of $\mathcal{H} \mathcal{R}_{\omega}$ are integers, we are going to see that the Harthong-Reeb line is equivalent to the system of limited real numbers $\left(\mathbb{R}_{l i m}, \simeq, \lesssim,+, \times\right)$ where $x \lesssim y$ means $(x \leq y)$ or $(x \simeq y)$.

Since, for every $X \in \mathcal{H} \mathcal{R}_{\omega}$ and $x \in \mathbb{R}_{\text {lim }}$ we have $X / \omega \in \mathbb{R}_{\text {lim }}$ and $\lfloor\omega x\rfloor \in \mathcal{H} \mathcal{R}_{\omega}$, we consider the two maps

$$
\left\{\begin{array}{rlll}
\varphi_{\omega}: \mathcal{H R}_{\omega} & \rightarrow & \mathbb{R}_{l i m} \\
X & \mapsto & X / \omega
\end{array}\right\} \text { and }\left\{\begin{array}{rlrl}
\psi_{\omega} & : & \mathbb{R}_{l i m} & \rightarrow \mathcal{H} \mathcal{R}_{\omega} \\
& x & \mapsto \omega x\rfloor
\end{array}\right\}
$$

While $\varphi_{\omega}$ is clearly additive ( $\mathbb{Z}$-linear), it is not the case for $\psi_{\omega}$. This is the source of some technical difficulties not always well treated in the litterature.

Then, for every $X, Y \in \mathcal{H} \mathcal{R}_{\omega}$ and $x \in \mathbb{R}_{\text {lim }}$, we have the following properties: 


$$
\begin{aligned}
& -X \leq_{\omega} Y \Rightarrow \varphi_{\omega}(X) \lesssim \varphi_{\omega}(Y) ; \\
& -\varphi_{\omega}\left(X+{ }_{\omega} Y\right) \simeq \varphi_{\omega}(X)+\varphi_{\omega}(Y) ; \\
& -\varphi_{\omega}\left(X \times_{\omega} Y\right) \simeq \varphi_{\omega}(X) \times \varphi_{\omega}(Y) ; \\
& -\varphi_{\omega}\left(0_{\omega}\right) \simeq 0 \text { and } \varphi_{\omega}\left(1_{\omega}\right) \simeq 1 ; \\
& -X={ }_{\omega} Y \quad \Leftrightarrow \quad \varphi_{\omega}(X) \simeq \varphi_{\omega}(Y) ; \\
& -\forall y \in \mathbb{R}_{l i m} \exists X \in \mathcal{H} \mathcal{R}_{\omega} \quad \varphi_{\omega}(X) \simeq y ; \\
& -\psi_{\omega} \circ \varphi_{\omega}(X)={ }_{\omega} X \text { and } \varphi_{\omega} \circ \psi_{\omega}(x) \simeq x .
\end{aligned}
$$

These properties are summarized by saying that $\varphi_{\omega}$ is an isomorphism from the system $\left(\mathcal{H} \mathcal{R}_{\omega},=_{\omega}, \leq_{\omega},+{ }_{\omega}, \times_{\omega}\right)$ to the system $\left(\mathbb{R}_{\text {lim }}, \simeq, \lesssim,+, \times\right)$ and that $\psi_{\omega}$ is the inverse isomorphism.

\section{The arithmetization of Euler scheme}

Since the two preceding systems are equivalent, it is theoretically possible to define on the Harthong-Reeb line an equivalent discrete notion for any continuous concept defined on $\mathbb{R}_{\text {lim }}$. For instance, each limited real number $a$ is represented by $\psi_{\omega}(a)=\lfloor\omega a\rfloor$ in $\mathcal{H} \mathcal{R}_{\omega}$. Similarly, a map $f: x \mapsto f(x)$ defined on a part of $\mathbb{R}_{\text {lim }}$ and with values in $\mathbb{R}_{\text {lim }}$ is represented by the map $F: X \mapsto F(X):=\lfloor\omega f(X / \omega)\rfloor$ defined on a part of $\mathcal{H} \mathcal{R}_{\omega}$ and with values in $\mathcal{H} \mathcal{R}_{\omega}$.

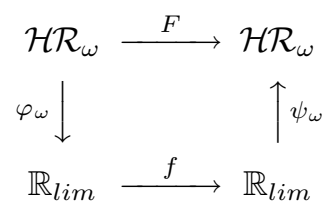

For a two variables map $g:(x, y) \mapsto g(x, y)$ the same process gives the discrete equivalent $G:(X, Y) \mapsto\lfloor\omega g(X / \omega, Y / \omega)\rfloor$.

The main disadvantage of this direct arithmetization method is that it is based on the real numbers on which the calculations are made before coming back to $\mathcal{H} \mathcal{R}_{\omega}$. On the contrary, our purpose is to stay in the discrete world of $\mathcal{H} \mathcal{R}_{\omega}$ without using any real numbers. A better idea for defining a discrete equivalent of a continuous object is to find an arithmetization of a construction method of this object. That is what we are going to do with the Euler scheme as a process for constructing a solution of a differential equation. This is a typical idea of Reeb who, at this time, was simply trying to make simulations of the moiré sensing on a personal computer.

\subsection{Arithmetization at the scale $\omega$}

We consider a function $x: t \mapsto x(t)$ which is a solution of a Cauchy problem

$$
\left\{\begin{array}{c}
x^{\prime}=f(t, x) \\
x(a)=b
\end{array}\right.
$$

where $f: U \rightarrow \mathbb{R}$ is a map $C^{1}$ defined on an open set $U$ of $\mathbb{R}^{2}$. We suppose that $a, b \in \mathbb{R}_{\text {lim }}, U \subset \mathbb{R}_{\text {lim }}^{2}$ and $f(U) \subset \mathbb{R}_{\text {lim }}$, and that $x$ is defined on an interval $[a, a+T]$ 
with $T \in \mathbb{R}_{l i m}$ and $T \not \chi 0$. We know that we can get a good approximation of the function $x$ by using the following Euler scheme with step $1 / \beta$

$$
\left\{\begin{array}{l}
t_{0}=a ; x_{0}=b \\
t_{n+1}=t_{n}+1 / \beta \\
x_{n+1}=x_{n}+1 / \beta f\left(t_{n}, x_{n}\right)
\end{array}\right.
$$

The real variables $t_{n}$ and $x_{n}$ are such that $x_{n}$ is an approximation of $x\left(t_{n}\right)$ and the error $\left|x\left(t_{n}\right)-x_{n}\right|$ is getting smaller when the step $1 / \beta$ of the method decreases to 0 . Thus, in our context, it is interesting to take $1 / \beta \simeq 0$. Moreover, since our goal is to find an equivalent scheme in $\mathcal{H} \mathcal{R}_{\omega}$ with integer variables, it is advantageous to assume that $\beta$ is a divisor of $\omega$. Finally, we suppose that

$$
\exists \alpha, \beta \in \mathbb{N} \quad \omega=\alpha \beta \text { and } \beta \simeq+\infty .
$$

Definition 7. An arithmetization of the initial Euler scheme (1) is a new iterative scheme with integer variables in $\mathcal{H} \mathcal{R}_{\omega}$

$$
\left\{\begin{array}{l}
T_{0}=A ; X_{0}=B \\
T_{n+1}=T_{n}+C \\
X_{n+1}=X_{n}+\Phi\left(\beta, T_{n}, X_{n}\right)
\end{array}\right.
$$

with $A, B, C \in \mathcal{H} \mathcal{R}_{\omega}$ and a function $\Phi$ such that, if we go back to $\mathbb{R}_{\text {lim }}$ using the real variables $t_{n}:=T_{n} / \omega$ and $x_{n}:=X_{n} / \omega$, we get a scheme infinitely close to (1), that is to say, an algorithm of the form

$$
\left\{\begin{array}{l}
t_{0}=a^{\prime} ; x_{0}=b^{\prime} \\
t_{n+1}=t_{n}+1 / \beta \\
x_{n+1}=x_{n}+1 / \beta f^{\prime}\left(t_{n}, x_{n}\right)
\end{array}\right.
$$

where $a^{\prime} \simeq a, b^{\prime} \simeq b$ and $f^{\prime}(t, x) \simeq f(t, x)$ for $t, x \in \mathbb{R}_{\text {lim }}$.

This definition establishes a strong link between the initial scheme (1) and its arithmetization (3). Let us now show how we can choose the components $A, B, C$ and $\Phi$ of (3). It is easy for the first three: $A:=\lfloor\omega a\rfloor, B:=\lfloor\omega b\rfloor^{5}$ and $C:=\alpha$. As an arithmetical translation of the term $1 / \beta f\left(t_{n}, x_{n}\right)$, it is quite natural to take

$$
\Phi\left(\beta, T_{n}, X_{n}\right):=\left\lfloor(1 / \beta)\left\lfloor\omega f\left(T_{n} / \omega, X_{n} / \omega\right)\right\rfloor\right\rfloor=F\left(T_{n}, X_{n}\right) \div \beta
$$

where $F\left(T_{n}, X_{n}\right):=\left\lfloor\omega f\left(T_{n} / \omega, X_{n} / \omega\right)\right\rfloor$ is an arithmetization of $f\left(t_{n}, x_{n}\right)$ and $\div$ denotes the arithmetic operation which gives the euclidian quotient. Of course, we suppose that the function $f$ is sufficiently simple so that its arithmetization $F$ is directly accessible in $\mathcal{H} \mathcal{R}_{\omega}$ without any calculus on $\mathbb{R}$. Thus, we consider the following scheme

$$
\left\{\begin{array}{l}
T_{0}=A ; X_{0}=B \\
T_{n+1}=T_{n}+\alpha \\
X_{n+1}=X_{n}+F\left(T_{n}, X_{n}\right) \div \beta
\end{array}\right.
$$

where $A:=\lfloor\omega a\rfloor, B:=\lfloor\omega b\rfloor$ and $F\left(T_{n}, X_{n}\right):=\left\lfloor\omega f\left(T_{n} / \omega, X_{n} / \omega\right)\right\rfloor$.

5. Of course, we suppose that these two elements are already known without any calculus in $\mathbb{R}$. This knowledge may be the result of a preceding arithmetization process. 
Proposition 6. If we add the condition $\alpha \simeq+\infty$ to the hypothesis (2), the scheme (4) is an arithmetization of the initial Euler scheme (1).

Proof. Since $\varphi_{\omega}$ is additive, it is sufficient to show that $\varphi_{\omega}\left(F\left(T_{n}, X_{n}\right) \div \beta\right)$ may be written $1 / \beta f^{\prime}\left(t_{n}, x_{n}\right)$ with the condition $f^{\prime}\left(t_{n}, x_{n}\right) \simeq f\left(t_{n}, x_{n}\right)$. Since

$$
\varphi_{\omega}\left(F\left(T_{n}, X_{n}\right) \div \beta\right)=\frac{1}{\omega}\left(\left\lfloor\frac{\left\lfloor\omega f\left(t_{n}, x_{n}\right)\right\rfloor}{\beta}\right\rfloor\right)
$$

we get

$$
\varphi_{\omega}\left(F\left(T_{n}, X_{n}\right) \div \beta\right)=\frac{1}{\omega}\left(\frac{\left\lfloor\omega f\left(t_{n}, x_{n}\right)\right\rfloor}{\beta}-\left\{\frac{\left\lfloor\omega f\left(t_{n}, x_{n}\right)\right\rfloor}{\beta}\right\}\right)
$$

then

$$
\varphi_{\omega}\left(F\left(T_{n}, X_{n}\right) \div \beta\right)=\frac{1}{\omega}\left(\frac{\omega f\left(t_{n}, x_{n}\right)}{\beta}-\frac{\left\{\omega f\left(t_{n}, x_{n}\right)\right\}}{\beta}-\left\{\frac{\left\lfloor\omega f\left(t_{n}, x_{n}\right)\right\rfloor}{\beta}\right\}\right)
$$

that is to say

$$
\varphi_{\omega}\left(F\left(T_{n}, X_{n}\right) \div \beta\right)=\frac{1}{\beta}\left(f\left(t_{n}, x_{n}\right)-\frac{\left\{\omega f\left(t_{n}, x_{n}\right)\right\}}{\omega}-\frac{1}{\alpha}\left\{\frac{\left\lfloor\omega f\left(t_{n}, x_{n}\right)\right\rfloor}{\beta}\right\}\right)
$$

Since $\omega$ and $\alpha$ are infinitely large, we get the result.

From now on, it is assumed that

$$
\exists \alpha, \beta \in \mathbb{N} \quad \omega=\alpha \beta, \alpha \simeq+\infty \text { and } \beta \simeq+\infty
$$

Under this hypothesis, we say that (4) is the arithmetization of (1) at the scale $\omega$.

\subsection{Interpretation at an intermediary scale}

We are now looking at the solution $\left(T_{n}, X_{n}\right)$ of (4). We would like to get an arithmetization of the initial function $t \mapsto x(t)$ as a discrete function $T \mapsto X(T)$ defined on an interval $[0, N]$ of $\mathbb{N}$. It appears that this solution $\left(T_{n}, X_{n}\right)$ is a sequence of points very distant from each other because $T_{n+1}-T_{n}=\alpha \simeq+\infty$. To get closer the points of this sequence, we can observe the solution at an intermediate scale.

A general principle of Reeb is that it is useful to work with two non trivial scales: the (strong) scale $\omega$ for computing the solution and a (lowest) scale $\omega^{\prime}$ with $1 \ll \omega^{\prime} \ll \omega$ for the graphical representation of the same solution.

Thus, we have to explain more formally what does it mean to go from the scale $\omega$ to another scale $\omega^{\prime}$. The natural idea is to perform an homothety followed by a truncation, i.e $X \mapsto\left\lfloor\frac{\omega}{\omega^{\prime}} X\right\rfloor$. This maps turns out to be equal to $\psi_{\omega^{\prime}} \circ \varphi_{\omega}: \mathcal{H R}_{\omega} \rightarrow \mathcal{H} \mathcal{R}_{\omega^{\prime}}$, so that we get the commutative diagram

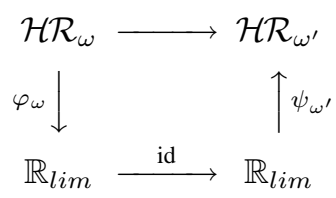


If $\omega^{\prime}$ is a divisor of $\omega$, that is to say if there is $\omega^{\prime \prime} \in \mathbb{N}$ such that $\omega=\omega^{\prime} \omega^{\prime \prime}$, we get the following nice form

$$
\forall X \in \mathcal{H R}_{\omega} \quad \psi_{\omega^{\prime}} \circ \varphi_{\omega}(X)=\left\lfloor\frac{X}{\omega^{\prime \prime}}\right\rfloor=X \div \omega^{\prime \prime}
$$

The question is to choose an interesting intermediate scale. Actually, we have already two intermediate scales: $\alpha$ and $\beta$ such that $\omega=\alpha \beta$. What is the best? If we choose $\beta$, then the second line in (4) is becoming better but the effect on the third line is not so good. If we choose $\alpha$, the third line is becoming better but not the second one. It appears that it is our interest to suppose that $\alpha=\beta$. Thus, we make a further assumption which is compatible with the previous (2) and (5)

$$
\exists \beta \in \mathbb{N} \quad \omega=\beta^{2} \text { and } \beta \simeq+\infty
$$

Hence, our intermediate scale is $\beta$ and going from the scale $\omega$ to the scale $\beta$ is just the map

$$
\begin{aligned}
\psi_{\beta} \circ \varphi_{\omega}: \mathcal{H R}_{\omega} & \longrightarrow \mathcal{H} \mathcal{R}_{\beta} \\
X & \longmapsto X \div \beta
\end{aligned}
$$

In order to get the interpretation of the scheme (4) at the intermediate scale $\beta$ we introduce the following notation: for every $Z \in \mathcal{H} \mathcal{R}_{\omega}, \widetilde{Z}:=Z \div \beta$ and $\widehat{Z}:=Z \bmod \beta$ respectively denotes the quotient and the remainder in the euclidean division of $Z$ by $\beta$. From the preceding explanation, we know that $Z=\widetilde{Z} \beta+\widehat{Z}$ with $\widetilde{Z} \in \mathcal{H} \mathcal{R}_{\beta}$ and $\widehat{Z} \in\{0, \ldots, \beta-$ $1\}$. Thus, for each $k, T_{k}=\widetilde{T}_{k} \beta+\widehat{T}_{k}, X_{k}=\widetilde{X}_{k} \beta+\widehat{X}_{k}$ and $F\left(T_{k}, X_{k}\right)=\widetilde{F}_{k} \beta+\widehat{F}_{k}$ with $\widetilde{T}_{k}, \widetilde{X}_{k}, \widetilde{F}_{k} \in \mathcal{H} \mathcal{R}_{\beta}$ et $\widehat{T}_{k}, \widehat{X}_{k}, \widehat{F}_{k} \in\{0,1, \ldots, \beta-1\}$.

From the relation $T_{n+1}=T_{n}+\beta$ we deduce that $T_{n+1} \div \beta=T_{n} \div \beta+1$, that is to say $\widetilde{T}_{n+1}=\widetilde{T}_{n}+1$. The equation $X_{n+1}=X_{n}+F\left(T_{n}, X_{n}\right) \div \beta$ becomes

$$
\widetilde{X}_{n+1} \beta+\widehat{X}_{n+1}=\widetilde{X}_{n} \beta+\widehat{X}_{n}+\widetilde{F}_{n}
$$

which is equivalent to the system

$$
\left\{\begin{array}{l}
\widetilde{X}_{n+1}=\widetilde{X}_{n}+\left(\widehat{X}_{n}+\widetilde{F}_{n}\right) \div \beta \\
\widehat{X}_{n+1}=\left(\widehat{X}_{n}+\widetilde{F}_{n}\right) \bmod \beta
\end{array}\right.
$$

Finally, we get the arithmetisation of Euler scheme (1) computed at the scale $\omega=\beta^{2}$ and interpreted at the intermediary scale $\beta$ :

$$
\left\{\begin{array}{l}
\widetilde{T}_{0}=A \div \beta, \widetilde{X}_{0}=B \div \beta \text { and } \widehat{X}_{0}=B \bmod \beta \\
\widetilde{T}_{n+1}=\widetilde{T}_{n}+1 \\
\widetilde{X}_{n+1}=\widetilde{X}_{n}+\left(\widehat{X}_{n}+\widetilde{F}_{n}\right) \div \beta \\
\widehat{X}_{n+1}=\left(\widehat{X}_{n}+\widetilde{F}_{n}\right) \bmod \beta
\end{array}\right.
$$

where $A=\left\lfloor\beta^{2} a\right\rfloor, B=\left\lfloor\beta^{2} b\right\rfloor$ and

$$
\begin{aligned}
\widetilde{F}_{n} & =F\left(\widetilde{T}_{n} \beta+A \bmod \beta, \widetilde{X}_{n} \beta+\widehat{X}_{n}\right) \div \beta \\
& =\left\lfloor\omega f\left(\frac{\widetilde{T}_{n} \beta+A \bmod \beta}{\omega}, \frac{\widetilde{X}_{n} \beta+\widehat{X}_{n}}{\omega}\right)\right\rfloor \div \beta .
\end{aligned}
$$


In the scheme (7), we have to understand that the $\widehat{X}_{k}$ are some auxiliary variables and that the $\widetilde{T}_{k}$ and the $\widetilde{X}_{k}$ are the relevant variables. Now, the set of pairs $\left(\widetilde{T}_{k}, \widetilde{X}_{k}\right)$ is the graph of a discrete function $T \mapsto X(T)$ defined on an interval $\mathbb{I}$ of $\mathbb{Z}$. We will say that this function is the arithmetisation of the initial function $t \mapsto x(t)$, computed at the scale $\beta^{2}$ and interpreted at the scale $\beta$.

\section{Examples of arithmetisation}

\subsection{The arithmetisation of the exponential function}

One of the first functions to which Reeb applied the previous arithmetization process was the exponential function.

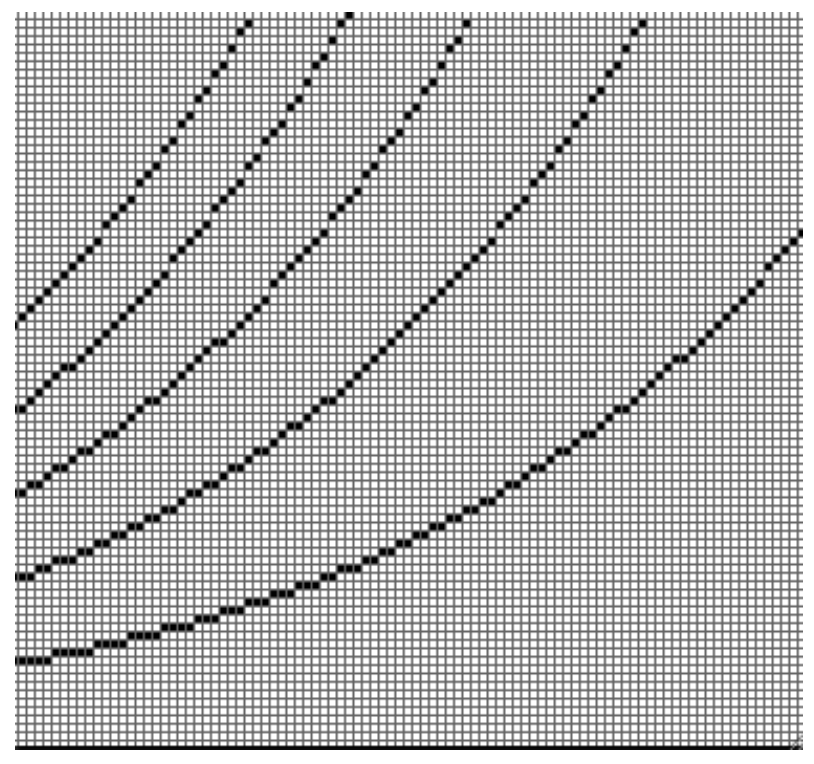

Figure 3. The arithmetisation of $t \mapsto \gamma e^{t}$ computed at the scale $\beta^{2}$ and interpreted at the scale $\beta$ for $\beta=50$ and $\gamma=0.2,0.4,0.6,0.8,1$.

The exponential function $x \mapsto e^{x}$ is the solution of the following Cauchy problem

$$
\left\{\begin{array}{cc}
x^{\prime} & = \\
x(0) & =1
\end{array}\right.
$$

The function $f$ of the general theory is now the projection $(t, x) \mapsto x$. Thus, we get

$$
F\left(\widetilde{T}_{n} \beta+A \bmod \beta, \widetilde{X}_{n} \beta+\widehat{X}_{n}\right)=\widetilde{X}_{n} \beta+\widehat{X}_{n} \text { and } \widetilde{F}_{n}=\widetilde{X}_{n}
$$


Since the initial condition is $x(0)=1$, we have $A=0$ et $B=\beta^{2}$. Consequently, the arithmetisation of the corresponding Euler scheme computed at the scale $\beta^{2}$ and interpreted at the scale $\beta$ is the following

$$
\left\{\begin{array}{l}
\widetilde{T}_{0}=0, \widetilde{X}_{0}=\beta \text { et } \widehat{X}_{0}=0 \\
\widetilde{T}_{n+1}=\widetilde{T}_{n}+1 \\
\widetilde{X}_{n+1}=\widetilde{X}_{n}+\left(\widetilde{X}_{n}+\widehat{X}_{n}\right) \div \beta \\
\widehat{X}_{n+1}=\left(\widetilde{X}_{n}+\widehat{X}_{n}\right) \bmod \beta
\end{array}\right.
$$

This is precisely the kind of algorithm proposed by G. Reeb. This algorithm defines a discrete function $T \mapsto E(T)$ for $T \geq 0$ in $\mathcal{H} \mathcal{R}_{\beta}$ which is an arithmetisation of the exponential function: $E(T) / \beta \simeq e^{T / \beta}$ for $T \in \mathcal{H} \mathcal{R}_{\beta}$ and $e^{t} \simeq E(\lfloor\beta t\rfloor) / \beta$ for $t \in \mathbb{R}_{\text {lim }}$.

\subsection{The arithmetisation of a straight line}

It may seem a strange idea to implement the previous aritmetization process on a function as elementary as $t \mapsto c t+d$. Yet, that was done by J.P. Reveillès with a surprising and intersecting result: a new approach to the notion of discrete line $[35,36,37,38,42$, 43].

We consider now a straight line $\Delta$ in the plane $\mathbb{R}^{2}$ with the equation $x=c t+d$ where $c$ and $d$ are two given elements of $\mathbb{R}_{\text {lim }}$. This line is the graph of the function $t \mapsto x(t):=c t+d$ which is the solution of the trivial Cauchy problem

$$
\left\{\begin{array}{ccc}
x^{\prime} & = & c \\
x(0) & = & d
\end{array}\right.
$$

The corresponding Euler scheme is

$$
\left\{\begin{array}{l}
t_{0}=0 \text { and } x_{0}=d \\
t_{n+1}=t_{n}+1 / \beta \\
x_{n+1}=x_{n}+c / \beta
\end{array}\right.
$$

and the arithmetisation of this last scheme, computed at the scale $\beta^{2}$ and interpreted at the scale $\beta$, is

$$
\left\{\begin{array}{l}
\widetilde{T}_{0}=0, \widetilde{X}_{0}=\left\lfloor\beta^{2} d\right\rfloor \div \beta \text { and } \widehat{X}_{0}=\left\lfloor\beta^{2} d\right\rfloor \bmod \beta \\
\widetilde{T}_{n+1}=\widetilde{T}_{n}+1 \\
\widetilde{X}_{n+1}=\widetilde{X}_{n}+\left(\widehat{X}_{n}+K\right) \div \beta \\
\widehat{X}_{n+1}=\left(\widehat{X}_{n}+K\right) \bmod \beta
\end{array}\right.
$$

where $K$ is the integer $\left\lfloor\beta^{2} c\right\rfloor \div \beta \in \mathcal{H} \mathcal{R}_{\beta}$. The set of pairs $\left(\widetilde{T}_{k}, \widetilde{X}_{k}\right)$ is the graph of a discrete function $T \mapsto X(T)$ defined on an interval $\mathbb{I}$ of $\mathbb{Z}$. This function is called the arithmetisation of the straight line $\Delta$, computed at the scale $\beta^{2}$ and interpreted at the scale $\beta$.

Proposition 7. For all $T \in \mathbb{I}$, we have $X(T)=\left\lfloor C_{\beta} T+D_{\beta}\right\rfloor$ where $C_{\beta}:=\frac{\left\lfloor\beta^{2} c\right\rfloor \div \beta}{\beta}$ and $D_{\beta}:=\frac{\left\lfloor\beta^{2} d\right\rfloor}{\beta}$. 
Proof. We introduce the variable $X_{n}:=\widetilde{X}_{n} \beta+\widehat{X}_{n}$ with values in $\mathcal{H} \mathcal{R} \omega$ where $\omega=\beta^{2}$. Then, we have $\widetilde{X}_{n}=X_{n} \div \beta$ and we get the inductive relation $X_{n+1}=X_{n}+K$. Thus, for each $n$ we have $X_{n}=X_{0}+n K$. Since $K=\lfloor\omega c\rfloor \div \beta$ and

$$
X_{0}=\widetilde{X}_{0} \beta+\widehat{X}_{0}=(\lfloor\omega d\rfloor \div \beta) \beta+\lfloor\omega d\rfloor \bmod \beta=\lfloor\omega d\rfloor
$$

we get the expression $\left.X_{n}=\right\rfloor n(\lfloor\omega c\rfloor \div \beta)+\lfloor\omega d\rfloor$ and finally

$$
\widetilde{X}_{n}=\left\lfloor\frac{n(\lfloor\omega c\rfloor \div \beta)+\lfloor\omega d\rfloor}{\beta}\right\rfloor .
$$

Since $\widetilde{T}_{n}=n$, the proof is done.

The elements of the graph of the function $T \mapsto X(T)$ are the points with integer coordinates which are on or just below the continuous straight line $\Delta_{\beta}$ with equation $x=C_{\beta} t+D_{\beta}$. The slope of $\Delta_{\beta}$ is $C_{\beta}=\frac{\left(\left\lfloor\beta^{2} c\right\rfloor \div \beta\right)}{\beta} \simeq c$ and for $t=0$ we have $x=D_{\beta}=\frac{\left\lfloor\beta^{2} d\right\rfloor}{\beta} \simeq \beta d$. Thus, for $d=0$ the line $\Delta_{\beta}$ is infinitely close to the initial line $\Delta$.

Moreover, the algorithm given by (10) is a variant of the famous Bresenham algorithm which is used for drawing a line in a discret plane like a computer screen. This fact shows that the algorithm provided by our arithmetization process is a good one. This property is interesting because this algorithm is given by a general and conceptual analysis while the Bresenham algorithm results of a specific work on a straight line.

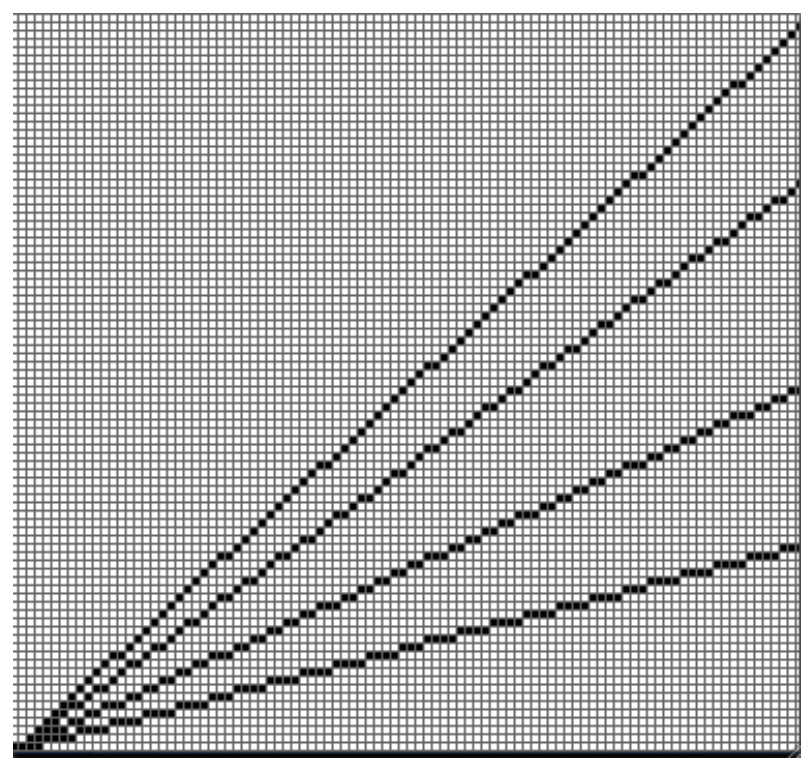

Figure 4. The arithmetisation of the line $15 y=a x$ computed at the scale $\beta^{2}$ and interpreted at the scale $\beta$ for $\beta=50$ and $a=4,7,11,14$. 
Finally, it is easy to check that the graph of the function $T \mapsto\left\lfloor C_{\beta} T+D_{\beta}\right\rfloor$ is the set of $(T, X) \in \mathbb{Z}^{2}$ such that

$$
0 \leq K T-\beta X+\beta D_{\beta} \leq \beta
$$

This kind of inequalities is at the origin of the concept of analytic discrete line introduced by Reveillès:

Given $a, b, \gamma, \tau \in \mathbb{Z}$ such that $0 \leq a \leq b, b \neq 0$ and $\tau>0$, the discrete analytic line with slope $(a, b)$, with thickness $\tau$ and origin $\gamma$ is the set of points $(X, Y) \in \mathbb{Z}^{2}$ such that

$$
\gamma \leq a X-b Y<\gamma+\tau
$$

This definition is at the beginning of a new scientific field of discrete geometry and graphical computer usually called discrete analytical geometry. It should be noted that, in these latest developments, nonstandard analysis is no longer used and the main theoretical tool seems to be arithmetic.

\section{Elements on the constructive content of the Harthong-Reeb line}

In the preceding sections, we have introduced a weak axiomatic version of nonstandard analysis which is enough to work on integer numbers. Then, we have defined the numerical system of the Harthong-Reeb line and the arithmetization process of the Euler scheme which allows us to construct an arithmetical analogous of a solution of some differential equations with variables in $\mathbb{R}$. Let us remark that the philosophy and the concept of the Harthong-Reeb line gave also rise to other kind of scientific developments, mainly around the works of Harthong $[14,15]$, developments which are not covered in the present paper.

A natural remark about what we have related is the constructive aspect of these system. In fact, once admitted the nonstandard language and the infinitely large natural number $\omega$ or $\beta$, we get a theory and a practice which seems relatively constructive: we can see that we only perform explicit calculus and reasoning without the use of any new transcendent principle. Furthermore, the nonstandard axiomatic we have chosen is as little transcendent as possible. Indeed, we only need the existence of some infinitely large number and we just did what is required for this purpose.

Of course, for the moment it is only an impression of constructivity and it may be noted at least two points which weaken this feeling:

- from the constructive point of view, the axiomatic introduction of infinitely large numbers is not completely satisfactory since the computational meaning of such entities is not given;

- there is no chance that the underlying logic used in the considered developments is completely conform to the strict constraints of intuitionnistic principles which are one of the ultimate criteria for the constructive mathematics.

More generally, this is the old debate about the constructivity of nonstandard analysis which arise again. On the one hand, the main criticism made against NSA is that this theory is highly non-constructive. According to this view, NSA and constructive mathematics occupies opposite positions in the geography of mathematics. For instance, the 
reaction of E. Bishop [3] (the master of constructive analysis) was to consider that NSA was the ultimate senseless mark of the non-constructive framework of classical analysis. One can also mention the judgement made by A. Connes on NSA [8]. The idea behind this type of criticism is that the typical nonstandard entities (like nonstandard numbers) are basically non-constructive, as fictitious as non-measurable set of Lebesgue theory or as the axiom of choice of set theory. Accordingly, theses entities would be nothing else that some non-essential artefacts of our formalism. On the other hand, Harthong and Reeb explains in [16] that, far from being an artefact, nonstandard analysis necessarily results of an intuitionnistic interpretation of the classical mathematical formalism (see also [41]). In a more concrete way, it is a common remark that the practice of NSA has a relatively constructive appearence. Moreover, some strange structural similarities have been noted between nonstandard and constructive proofs [44]. Finally, thanks to the works of P. Martin-Löf [26], I. Moerdijk [27] and E. Palmgren [30, 31], there are now new presentations of nonstandard analysis which completely fit with the constructive constraints (see also [5]).

Thus, it is now time for us to move beyond a sterile polemic and to begin the conceptual analysis of the question of the constructivity of the Harthong-Reeb line $\mathcal{H} \mathcal{R}{ }_{\omega}$. In a recent work [13], we have shown that $\mathcal{H} \mathcal{R}_{\omega}$, just slightly modified, satisfies in a non-trivial way the Bridges axioms of a constructive line. These axioms defined a kind of abstract structure which may be called a Bridges-Heyting field and which concentrates the essential characteristics of the continuous line from the constructive point of view; then, using only intuitionnistic logic, all the known constructive properties of this line may be derived. In our work on $\mathcal{H} \mathcal{R}_{\omega}$, starting with accurate definitions of $=_{\omega}$ and $>_{\omega}$ and using a special logical framework closed to the intuitionnistic one, we prove that $\mathcal{H} \mathcal{R}_{\omega}$ is a Bridges-Heyting field. We believe that the constructive content of this version of $\mathcal{H} \mathcal{R}_{\omega}$ is measured by the quality of our logical framework. This last one can be summed up in three rules: 1) the usual relation $=$ and $>$ are decidable on integers (limited or nonlimited), 2) the usual constructive integers are identified to the limited one and 3) we use only intuitionnistic logic. Conversely, the lack of constructivity of $\mathcal{H} \mathcal{R}_{\omega}$ should also be read in this logical context: it is clear that the combination of rules 1) and 2) is not totally satisfactory because it is only for the usual integers that the relations $=$ and $>$ are unquestionably decidable.

This last restriction on the constructivity of $\mathcal{H} \mathcal{R}_{\omega}$ is clearly linked to the axiomatic way we chose for the introduction of infinitely large numbers. More generally, it is now well known that the axioms are a real obstacle in computational mathematics. From this viewpoint, it is always better to replace a problematic axiom by a roughly equivalent computational rule $[11,12]$. This is the direction in which we are currently working with the hope to get a more constructive version of the Harthong-Reeb line. For this purpose, we are partly using the idea of Laugwitz and Schmieden on infinitely large natural numbers $[19,21,20]$ and the framework of Martin-Löf type theory [24, 25, 26]. We believe that, for a system like the Harthong-Reeb, it would be possible to obtain a sufficiently constructive structure so that the proofs written in it may be translated into exacts programs.

\section{Appendix on external induction}

We now supplement our minimal form of nonstandard analysis by the introduction of a special induction that fits with external formulae. This inductive defining principle is our last axiom: 
LIM5. (External inductive defining principle): Let $p$ a limited natural number and $\mathcal{P}(U)$ be an internal or an external formula for which $U$ denotes an arbitrary finite sequence in $\mathbb{Z}^{p}$. We suppose that:

1) there exists an element $X_{0} \in \mathbb{Z}^{p}$ such that $\mathcal{P}\left(\left(X_{0}\right)\right)$;

2) for every limited $n \in \mathbb{N}$ and for every sequence $\left(X_{k}\right)_{0 \leq k \leq n}$ in $\mathbb{Z}^{p}$ such that $\mathcal{P}\left(\left(X_{k}\right)_{0 \leq k \leq n}\right)$, there exists $X_{n+1} \in \mathbb{Z}^{p}$ such that we have $\mathcal{P}\left(\left(X_{k}\right)_{0 \leq k \leq n+1}\right)$.

Therefore, there exists an internal infinite sequence $\left(X_{k}\right)_{k \in \mathbb{N}}$ in $Z^{p}$ such that, for all standard $n \in N, \mathcal{P}\left(\left(X_{k}\right)_{0 \leq k \leq n}\right)$.

This principle means that the sequence of values $X_{k}$ for $k$ limited can be extended in a complete sequence $\left(X_{k}\right)_{k \in \mathbb{N}}$ defined for all naturel numbers $k$. Saying that this sequence is internal means that it has all the properties of the classical sequences in usual number theory. Particularly, if $\mathcal{Q}(X)$ is an internal formula, then the collection $\left\{k \in \mathbb{N} ; \mathcal{Q}\left(X_{k}\right)\right\}$ is an internal subset of $\mathbb{N}$.

\section{References}

[1] Barreau H., Harthong J. (Editeurs), "La Mathématique non standard: histoire, philosophie, dossier scientifique”, Editions du CNRS, Paris, 1999.

[2] BISHOP E., "Foundations of Constructive Analysis", McGraw-Hill, 1967.

[3] Bishop E., "Review of Foundation of Infinitesimal Calculus of Keisler H.J.", Bulletin of American Math. Soc., vol. 83, (1977) 205-207.

[4] Bishop E., BRIDGEs D., "Constructive Analysis”, Springer-Verlag, 1985.

[5] Boldini P., "La priorité de la preuve : le cas de la signification non-standard des constantes logiques”, Revue Internationale de Philosophie, vol. 230, 2004, 437-447.

[6] Bridges D., ReEves S., "Constructive Mathematics, in Theory and Programming Practice", Centre for Discrete Mathematics and Theorical Computer Science, CDMTCS-068, november1997.

[7] BRIDges D., "Constructive Mathematics: A Foundation for Computable Analysis", Theor. Comput. Sci., vol. 219, num. 1-2, 1999, 95-109.

[8] Connes A., "Noncommutative Differential Geometry and the Structure of Space-Time", in The Geometric Universe, HuggetT S.A., MAson L.J., ToD K.P Editors, Oxford University Press, 1998, 49-80.

[9] Diener F., Reeb G., Analyse Non Standard, Hermann, Paris, 1989.

[10] Diener M., "Application du Calcul de Harthong-Reeb aux Routines Graphiques, in Le Labyrinthe du Continu, 1992, 424-435.

[11] DoweK G., "La théorie des types et les systèmes informatiques de traitement des démonstrations mathématiques", Mathématiques et Science Humaines / Mathematical Social Sciences, vol. $165,2004,13-29$.

[12] DoweK G., Les Métamorphoses du calcul, Editions Le Pommier, 2007.

[13] Fuchs L., Largeteau-Skapin G., Wallet G., Andres E., Chollet A.", “A first look into a formal and constructive approach for discrete geometry using nonstandard analysis", in Discret Geometry for Computer Imagery, Lecture Notes in Computer Science, vol. 4992, Springer Berlin / Heidelberg, 2008, 21-32.

[14] Harthong J., "Éléments pour une théorie du continu", Astérisque, vol. 109/110, 1983, 235-244. 
[15] Harthong J., "Une théorie du continu", in [1], 1989, 307-329.

[16] Harthong J., ReEb G., "Intuitionnisme 84”, in [1], 1989, 213-252.

[17] Holin H., "Harthong-Reeb analysis and digital circles", The Visual Computer, vol. 8, num. 1, 1991, 8-17.

[18] Holin H., "Some Artefacts of Integer-computer Circles", Ann. Math. Artif. Intell., vol. 16, 1996,153-181.

[19] Laugwitz D., Schmieden C., "Eine Erweiterung der Infinitesimalrechnung”, Mathematische Zeitschrift, vol. 89, 1958,1-39.

[20] Laugwitz D., "Leibniz' Principle and Omega Calculus”, in [40], 1992, 144-155.

[21] LAUGWITZ D., " $\Omega$-calculus as a generalization of field extension", in Nonstandard Analysis - Recent developments, HURD A. editor, Lecture notes in Mathematics, Springer, Berlin, 1983, 144-155.

[22] LOBRY C., “Et pourtant ... ils ne remplissent pas $\mathbb{N} ”$, Aléas, Lyon, 1989.

[23] Lutz R., "La force modélisatrice des théories infinitésimales faibles", in [40], 1992, 414-423.

[24] MARTIN-LÖF P., "Constructive mathematics and computer programming", In Logic, Methodology and Philosophy of Science VI, North-Holland, 1980, 153-175.

[25] MARTIN-LÖF P., “Intuitionnistic Type Theory”, Bibliopolis, Napoli, 1984.

[26] Martin-LÖF P., "Mathematics of infinity", COLOG-88 Computer Logic, Lecture Notes in Computer Science, Springer-Verlag, 1990, 146-197.

[27] Moerdik I., "A model for intuitionnistic non-standard arithmetic", Annals of Pure an Applied Logic, vol. 73, 1995, 37-51.

[28] Nelson E., "Internal Set Theory: A new approach to nonstandard analysis", Bulletin of the American Mathematical Society, vol. 83, num. 6, 1977, 1165-1198.

[29] Nelson E., "Radically Elementary Theory", Princeton University Press, Annals of Mathematics Studies, 1987.

[30] Palmgren E., "A constructive approach to nonstandard analysis", Annals of Pure and Applied Logic, vol. 73, 1995, 297-325.

[31] Palmgren E., "Developments in Constructive Nonstandard Analysis", The Bulletin of Symbolic Logic, vol. 4, num. 3, 1998, 233-272.

[32] REEB G., "La mathématique non standard vieille de soixante ans ?", Cahiers de Topologie et de Géométrie Différentielle, vol. 22, 1981, 149-154.

[33] REEB G., “0, 1, 2, etc... ne remplissent pas (du tout) $\mathbb{N}$ ”, in [9], 1989, 151-158.

[34] Reeb G., Reveillès J.P., Troesch A., Urlacher E., "Equations différentielles en nombres entiers", Rapport de recherche, IRMA, Strasbourg, 1987.

[35] ReVeillès J.P., "Les paliers des droites de Bresenham”, in Pixim 88, Hermès, 1988, 81-101.

[36] Reveillès J.P., "Géométrie discrète, Calcul en nombres entiers et algorithmique”, Université Louis Pasteur, Strasbourg, 1991.

[37] ReVEILlÈs J.P., "Mathématiques discrètes et analyse non standard, in [40], 1992.

[38] Reveillès J.P., Richard D., "Back and Forth between continuous and discrete for the working computer scientist", Annals of Mathematics and Artificial Intelligence, Mathematics and Informatic, vol. 16, Èd. Baltzer, num. 1-4, Suisse, 1996, 89-152.

[39] Robinson A., "Non-standard analysis", 2nd ed, American Elsevier, New York, 1974.

[40] Salanskis J-M., SinaCeurs H. editors, "Le Labyrinthe du Continu”, Springer, 1992.

[41] SAlansKis J-M.", "Le constructivisme non standard", Presses Universitaires du Septentrion, Histoire des sciences, 1999. 
[42] Troesch A., "Interprétation géométrique de l'algorithme d'Euclide et reconnaissance de segments", Theor. Comput. Sci., vol. 115, num. 2, 1993, 291-319.

[43] Troesch A., "Droite discrètes et calendriers", Mathématiques et Sciences Humaines / Mathematics and Social Sciences, vol. 141, 1998, 11-41.

[44] Wattenberg F., "Nonstandard Analysis and Constructivism?", Studia Logica, Springer Netherland, vol. 47, num. 3, 1988, 303-309. 